\title{
Positivity-Preserving Numerical Methods for Belousov-Zhabotinsky Reaction
}

\author{
Yuro Adachi ${ }^{1}$, Novrianti ${ }^{1 *}$, Okihiro Sawada ${ }^{2}$ \\ ${ }^{1}$ Applied Physics Course, Faculty of Engineering, Gifu University, Gifu, Japan \\ ${ }^{2}$ Faculty of Engineering, Kitami Institute of Technology, Kitami, Japan \\ Email: *x3912006@edu.gifu-u.ac.jp
}

How to cite this paper: Adachi, Y., Novrianti and Sawada, O. (2020) PositivityPreserving Numerical Methods for BelousovZhabotinsky Reaction. Applied Mathematics, 11, 943-950.

https://doi.org/10.4236/am.2020.1110061

Received: August 28, 2020

Accepted: October 6, 2020

Published: October 9, 2020

Copyright (C) 2020 by author(s) and Scientific Research Publishing Inc.

This work is licensed under the Creative Commons Attribution International License $(\mathrm{CC}$ BY 4.0).

http://creativecommons.org/licenses/by/4.0/

\begin{abstract}
The existence of positive solutions to the system of ordinary differential equations related to the Belousov-Zhabotinsky reaction is established. The key idea is to use a new successive approximation of solutions, ensuring its positivity. To obtain the positivity and invariant region for numerical solutions, the system is discretized as difference equations of explicit form, employing operator splitting methods with linear stability conditions. Algorithm to solve the alternate solution is given.
\end{abstract}

\section{Keywords}

Positive Solution, Difference Equation, Belousov-Zhabotinsky Reaction, Invariant Region, Maximum Principle

\section{Introduction}

The goal is to give new discretization scheme for some reaction diffusion equations, which a priori ensures the positivity-preserving. The model equations incorporate a modified version of Holling-Tanner (or Leslie-Gower) functional response, which are studied for pattern dynamics and Turing instability. We consider the reaction diffusion equations of Keener-Tyson model for Belousov-Zhabotinsky reaction:

$$
\left\{\begin{array}{l}
\partial_{t} u=\Delta u+u(1-u) / \varepsilon-h v(u-q) /(u+q), \\
\partial_{t} v=d \Delta v-v+u, \\
\left.u\right|_{t=0}=u_{0},\left.v\right|_{t=0}=v_{0} .
\end{array}\right.
$$

See [1] and [2]. Here, $u=u(x, t)$ and $v=v(x, t)$ stand for the unknown scalar functions at $x \in \mathbb{R}^{n}$ and $t>0$ which denote the concentrations in a vessel of $\mathrm{HBrO}_{2}$ and $\mathrm{Ce}^{4+}$, respectively. Besides, $u_{0}=u_{0}(x)$ and $v_{0}=v_{0}(x)$ are given non-negative functions as the initial data. Throughout of this paper, to avoid the difficulty arising from boundaries, the Cauchy problem is treated in the whole space $\mathbb{R}^{n}$ for 
$n \in \mathbb{N}$ or, the periodic boundary conditions are assumed in a bounded domain. In [1], an example of constants is concretely listed-up as $\varepsilon \approx 0.032, q \approx 2.0 \times 10^{-4}$ and $d \approx 0.6 \times \varepsilon ; h:=\rho / \varepsilon$ and $\rho \approx 1 / 2$ stands for the excitability which governs dynamics of a pattern formulation. It has been used the notation of differentiation; $\partial_{t}:=\partial / \partial t$, $\Delta:=\sum_{i=1}^{n} \partial_{i}^{2}, \partial_{i}:=\partial / \partial x_{i}$ for $i=1, \ldots, n$.

On (BZ), the positivity of $u$ is a critical issue. In [3], the time-global existence of positive unique smooth solutions to (BZ) was established. They also obtained that $\mathbb{R}_{+}^{2}$ and $S:=(q, \bar{u})^{2}$ are invariant regions, where $\bar{u} \in(q, 1)$ is a unique root of

$$
u(1-u)(u+q)-\varepsilon h q(u-q)=0 .
$$

That is to say, when the initial data $\left(u_{0}, v_{0}\right) \in S$, the solution $(u, v) \in$ $S$ for $t>0$. The aim of this paper is to establish the similar results for numerical solutions to the difference equations of discretized (BZ) of special type. In particular, our numerical scheme congenitally equips the positivy-preserving and the maximum principle.

The key idea in [3] is to use the following successive approximation:

$$
\begin{aligned}
\partial_{t} u_{\ell+1} & =\Delta u_{\ell+1}+u_{\ell}\left(1-u_{\ell+1}\right) / \varepsilon-h v_{\ell}\left(u_{\ell+1}-q\right) /\left(u_{\ell}+q\right), \\
\partial_{t} v_{\ell+1} & =d \Delta v_{\ell+1}-v_{\ell+1}+u_{\ell},
\end{aligned}
$$

for $\ell \in \mathbb{N}$ with $\left.u_{\ell+1}\right|_{t=0}=u_{0}$ and $\left.v_{\ell+1}\right|_{t=0}=v_{0}$, starting at $u_{1}:=e^{t \Delta} u_{0}$ and $v_{1}:=e^{-t} e^{t \Delta} v_{0}$ with non-negative initial data $u_{0}$, $v_{0} \in B U C\left(\mathbb{R}^{n}\right)$. Here, $B U C$ is the space of all bounded uniformly continuous functions. The virtue of this scheme is to be ensured that $u_{\ell} \geq 0$ and $v_{\ell} \geq 0$ for all $\ell \in \mathbb{N}$, automatically. So, one can obtain the time-local non-negative classical solutions to (BZ) as $u=\lim _{\ell \rightarrow \infty} u_{\ell}$, $v=\lim _{\ell \rightarrow \infty} v_{\ell}$. We would emphasize that this technique can be applicable to construct positive (or, non-negative) solutions to ordinary differential equations and positive numerical solutions to finite difference equations.

There are many literatures on structure-preserving numerical methods for partial differential equations; e.g. [4] and references therein. Moreover, the researchers on reaction diffusion equations often discuss the positivity of numerical solutions, when the time-step size $\Delta t$ is small enoughly; the reader can find it in e.g. [5]. In this paper, we introduce a new difference scheme which produces positive numerical solutions for arbitrary large $\Delta t$; for the partial difference equations, we additionally impose the linear stability condition. From this view point, it has been known that Mimura and his collaborators obtained the positive numerical solutions to the system of some reaction diffusion equations; see ( [6], [7]). However, it seems to be new that a numerical scheme leads us to invariant regions.

In addition, there are many numerical results on $\mathrm{BZ}$ reaction; e.g. [8] and references therein. On the other hand, our scheme is an explicit method which has the features as aiming at application to validated numerics, in the future.

This paper is organized as follows. In Section 2, the extension of problem in ordinary differential equation is given, and we shall prove the existence of positive solutions to the system of ordinary differential equations as the result. To ensure the existence of numerical solution$\mathrm{s}$, in Section 3 we will be devoted to give a scheme of the difference equations which produce positive numerical solutions, automatically. We will state results for an invariant region of these numerical solutions to the discretized (BZ) in Section 4, by employing the operator 
splitting methods, and for the end we give the conclusion in Section 5.

\section{Ordinary Differential Equations}

In this section, the construction of time-local positive solutions to the system of first order ordinary differential equations (ODE) is discussed. Let $m \in \mathbb{N}$, we deal with the following system of nonlinear ODE:

$$
u_{i}^{\prime}=-f_{i}(\mathbf{u}) u_{i}+g_{i}(\mathbf{u}), \quad t>0, \quad u_{i}(0)=a_{i}, \quad i=1, \ldots, m .
$$

Throughout this paper, for simplicity of notation, $t=0$ is the initial time, ${ }^{\prime}:=d / d t, \mathbf{u}:=\left(u_{1}, \ldots, u_{m}\right)$. Here, $u_{i}=u_{i}(t)$ are unknown functions for $t>0$ and $i=1, \ldots, m$. Besides, $a_{i} \geq 0$ are given initial data, $f_{i} \geq 0$ and $g_{i} \geq 0$ are also given function. We often rewrite $(\mathrm{P})$ into the following vector valued ODE:

$$
\mathbf{u}^{\prime}=-F(\mathbf{u}) \mathbf{u}+\mathbf{g}(\mathbf{u}), \quad t>0, \quad \mathbf{u}(0)=\mathbf{a} .
$$

Here, we have denoted $\mathbf{a}:=\left(a_{1}, \ldots, a_{m}\right), \mathbf{g}:=\left(g_{1}, \ldots, g_{m}\right)$, and $F$ is the diagonal $m \times m$ matrix whose $(i, i)$-component is $f_{i}$.

When $m=2, u:=u_{1}, v:=u_{2}, a_{1}:=u_{0}, a_{2}:=v_{0}$ and

$$
F:=\left(\begin{array}{cc}
u / \varepsilon+h v /(u+q) & 0 \\
0 & 1
\end{array}\right), \quad \mathbf{g}:=\left(\begin{array}{c}
u / \varepsilon+h q v /(u+q) \\
u
\end{array}\right)
$$

are taken, then $(\mathrm{P})$ is equivalent to the uniform-in-space (BZ). The model problem $(\mathrm{P})$ is often used to describe dynamics of nonlinear chemical or biological systems, for example, the Lotka-Volterra type equations of predator-prey models with density-dependent inhibition (Holling's type II or type IV), epidemic SIV (or, SHIV) models and the Gierer-Meinhardt model. We especially treat fractional nonlinear terms, and the denominator takes on the value of zero for negative solutions. Hence, the positivity of solutions to $(\mathrm{P})$ is strongly required, and so is even in its approximation.

We shall state the main results in this paper.

Theorem 1. If $f_{i}, g_{i} \geq 0$ are local Lipschitz continuous and $a_{i} \geq 0$, then there exists a time-local unique solution $u_{i} \geq 0$ to $(\mathrm{P})$.

Proof. Let $a_{i} \geq 0$ for $i=1, \ldots, m$. For the sake of simplicity, let us assume that $\mathbf{a} \neq \mathbf{0}$ and $g_{i}(\mathbf{v})>0$ for $\mathbf{v} \neq \mathbf{0}$. Making the approximation sequences $\left\{u_{i}^{\ell}\right\}_{\ell=1}^{\infty}$ for $i=1, \ldots, m$, we begin with $\mathbf{u}^{1}(t):=\mathbf{a}$ for $t \geq 0$ For each $\ell \in \mathbb{N}$, we successively define $\mathbf{u}^{\ell+1}$ as the solution to the system of linear non-autonomous ODE:

$$
\left(\mathbf{u}^{\ell+1}\right)^{\prime}=-F\left(\mathbf{u}^{\ell}\right) \mathbf{u}^{\ell+1}+\mathbf{g}\left(\mathbf{u}^{\ell}\right), \quad t>0, \quad \mathbf{u}^{\ell+1}(0)=\mathbf{a}
$$

with vectors of non-negative $\mathbf{u}^{\ell}$ and $\mathbf{a}$. So, $(\mathrm{SA})$ is equivalent to the integral equation

$$
\mathbf{u}^{\ell+1}(t)=\mathbf{a}-\int_{0}^{t} F\left(\mathbf{u}^{\ell}(s)\right) \mathbf{u}^{\ell+1}(s) d s+\int_{0}^{t} \mathbf{g}\left(\mathbf{u}^{\ell}(s)\right) d s .
$$

Heuristically, if $F$ is a constant matrix, then $\mathbf{v}^{\prime}=-F \mathbf{v}, t>0, \mathbf{v}(0)=$ a admits a solution $\mathbf{v}(t)=e^{-F t} \mathbf{a}$. In this situation, we thus have

$$
\mathbf{u}^{\ell+1}(t)=e^{-F t} \mathbf{a}+\int_{0}^{t} e^{-F(t-s)} \mathbf{g}\left(\mathbf{u}^{\ell}(s)\right) d s .
$$


For general matrix-valued functions $F$, one may construct $\mathbf{u}^{\ell+1}$ for each $\ell \in \mathbb{N}$ by perturbation theory, at least time-locally.

Obviously, $u_{i}^{1} \geq 0$ for $i=1, \ldots, m$ and $\left\|\mathbf{u}^{1}(t)\right\|=\|\mathbf{a}\|$ for $t \geq 0$. Here, we have used the $\max$ norm for vectors $\|\mathbf{v}\|:=\max _{i=1, \ldots, m}\left|v_{i}\right|$ for $\mathbf{v}:=\left(v_{1}, \ldots, v_{m}\right)$, as well as to matrices $\|F\|:=\max _{i, j=1, \ldots, m}\left|f_{i j}\right|$ for $F:=\left(f_{i j}\right)$. In what follows, we will show the positivity and boundedness of $u_{i}^{\ell+1}$ by induction in $\ell$. For $\mathbf{u}^{2}$, it holds true that

$$
\begin{aligned}
\left\|\mathbf{u}^{2}(t)\right\| & \leq\|\mathbf{a}\|+\int_{0}^{t}\left\|F\left(\mathbf{u}^{1}(s)\right)\right\| \cdot\left\|\mathbf{u}^{2}(s)\right\| d s+\int_{0}^{t}\left\|\mathbf{g}\left(\mathbf{u}^{1}(s)\right)\right\| d s \\
& \leq\|\mathbf{a}\|+\|F(\mathbf{a})\| \cdot t \cdot \max _{0 \leq s \leq t}\left\|\mathbf{u}^{2}(s)\right\|+t \cdot\|\mathbf{g}(\mathbf{a})\| .
\end{aligned}
$$

Taking $\max _{0 \leq t \leq \tau}$ in both hand side, we have

$$
\left\|\mathbf{u}^{2}(t)\right\| \leq 2\|\mathbf{a}\| \quad \text { for } \quad t \in\left[0, T_{2}\right]
$$

with $T_{2}:=\min \{1 /(3\|F(\mathbf{a})\|),\|\mathbf{a}\| /(3\|\mathbf{g}(\mathbf{a})\|)\}$. In addition, we can also obtain that $u_{i}^{2} \geq 0$. Indeed, let us assume that there exists a $t_{*} \in\left(0, T_{2}\right]$ such that $u_{i}^{2}\left(t_{*}\right)=0$ for some $i=1, \ldots, m$. Without loss of generality, $t_{*}$ is the first time when $u_{i}^{2}$ touches 0 . So, at $t_{*}$, we see that $\left(u_{i}^{2}\right)^{\prime} \leq 0, f_{i}\left(\mathbf{u}^{1}\right) u_{i}^{2}=0$, and $g_{i}\left(\mathbf{u}^{1}\right)>0$. This contradicts to the fact that $\mathbf{u}^{2}$ is a solution to $(\mathrm{SA})$ with $\ell=1$.

Let $\ell \geq 2$. Assume that $\left\|\mathbf{u}^{\ell}(t)\right\| \leq 2\|\mathbf{a}\|$ and $u_{i}^{\ell}(t) \geq 0$ hold for $t \in\left[0, T_{0}\right]$ and $i=1, \ldots, m$, where $T_{0}>0$ will be determined later. We now argue on $\mathbf{u}^{\ell+1}$. By assumption, it is easy to see that

$$
\begin{aligned}
\left\|\mathbf{u}^{\ell+1}(t)\right\| & \leq\|\mathbf{a}\|+\int_{0}^{t}\left\|F\left(\mathbf{u}^{\ell}(s)\right)\right\| \cdot\left\|\mathbf{u}^{\ell+1}(s)\right\| d s+\int_{0}^{t}\left\|\mathbf{g}\left(\mathbf{u}^{\ell}(s)\right)\right\| d s \\
& \leq\|\mathbf{a}\|+M_{f} \cdot t \cdot \max _{0 \leq s \leq t}\left\|\mathbf{u}^{\ell+1}(s)\right\|+t \cdot M_{g} \\
& \leq 2\|\mathbf{a}\| \quad \text { for } \quad t \in\left[0, T_{0}\right]
\end{aligned}
$$

with $T_{0}:=\min \left\{1 /\left(3 M_{f}\right),\|\mathbf{a}\| /\left(3 M_{g}\right)\right\}$, where

$$
M_{f}:=\sup _{\|\mathbf{v}\| \leq 2\|\mathbf{a}\|, \mathbf{v} \geq \mathbf{0}}\|F(\mathbf{v})\|, \quad M_{g}:=\sup _{\|\mathbf{v}\| \leq 2\|\mathbf{a}\|, \mathbf{v} \geq \mathbf{0}}\|\mathbf{g}(\mathbf{v})\| .
$$

In addition, we can also see that $u_{i}^{\ell+1} \geq 0$ for $i$ by the same contradiction argument above. This means that $\left\|\mathbf{u}^{\ell}(t)\right\| \leq 2\|\mathbf{a}\|$ and $u_{i}^{\ell} \geq 0$ hold for all $\ell \in \mathbb{N}$ and $i=1, \ldots, m$ for $t \in\left[0, T_{0}\right]$.

It is straightforward to get the continuity of solutions. One may also see that $\left\{\mathbf{u}_{\ell}\right\}_{\ell=1}^{\infty}$ is a Cauchy sequence in $C\left(\left[0, T_{0}\right] ; \mathbb{R}^{m}\right)$. So, the $\operatorname{limit}\left(u_{1}(t), \ldots, u_{m}(t)\right)=\mathbf{u}(t)=\lim _{\ell \rightarrow \infty} \mathbf{u}^{\ell}(t)$ exists for $t \in\left[0, T_{0}\right]$, and satisfies $(\mathrm{P}) ; u_{i}(t) \geq 0$ for $i=1, \ldots, m$ by construction. The uniqueness follows from Gronwall's inequality, directly.

Note that the proof is easy, if $a_{i}>0$ for all $i$. In Theorem 1 , it is not needed to use neither the existence of stable solutions to $(\mathrm{P})$, comparison principle, nor a priori estimates by Lyapunov functions.

\section{Difference Equations}

We will argue the numerical algorithm for positive solutions. We first discuss a discretization of $(\mathrm{P})$. To obtain positive solutions, our proposal is to choose the following difference equations, mixing the forward and backward Euler methods:

$$
\frac{\mathbf{u}^{k+1}-\mathbf{u}^{k}}{\Delta t}=-F\left(\mathbf{u}^{k}\right) \mathbf{u}^{k+1}+\mathbf{g}\left(\mathbf{u}^{k}\right), \quad k \in \mathbb{N}_{0}:=\mathbb{N} \cup\{0\},
$$


where $\mathbf{u}^{k}=\left(u_{1}^{k}, \ldots, u_{m}^{k}\right), t_{k}:=k \Delta t$ for $\Delta t>0$ and $u_{i}^{0}=a_{i} \geq 0$ for $i=1, \ldots, m$. Clearly, (DE) is a mimic of (SA). In addition, we obviously see that the numerical solution $\mathbf{u}^{k}$ to (DE) tends to the solution $\mathbf{u}(t)$ to $(\mathrm{P})$ at $t=t_{k}$ for each $k$ as $\Delta t \rightarrow 0$.

Theorem 2. If $f_{i}, g_{i} \geq 0$ are Lipschitz continuous, $\Delta t>0$ and $u_{i}^{0} \geq 0$, then the numerical solution $u_{i}^{k} \geq 0$ to (DE) exists for $k \in \mathbb{N}$.

Proof of Theorem 2. We rewrite (DE) into the explicit form as

$$
u_{i}^{k+1}=\frac{u_{i}^{k}+g_{i}\left(\mathbf{u}^{k}\right) \Delta t}{1+f_{i}\left(\mathbf{u}^{k}\right) \Delta t}, \quad k \in \mathbb{N}_{0}, \quad i=1, \ldots, m .
$$

So, $u_{i}^{k+1} \geq 0$, if $u_{i}^{k} \geq 0$. Thus, one can prove it by induction.

The advantage of Theorem 2 is that we may take arbitrary large $\Delta t$.

The spirit of (DE) is still valid on the numerical methods for construction of non-negative solutions to the partial differential equations. For simplicity, let $n=1$, and consider the discretization $\mathbf{u}_{j}^{k}$ of $\mathbf{u}\left(x_{j}, t_{k}\right)$ for $x_{j}:=j \Delta x$ and $t_{k}:=k \Delta t$ satisfying

$$
\frac{\mathbf{u}_{j}^{k+1}-\mathbf{u}_{j}^{k}}{\Delta t}=d \frac{\mathbf{u}_{j+1}^{k}-2 \mathbf{u}_{j}^{k}+\mathbf{u}_{j-1}^{k}}{\Delta x^{2}}-F\left(\mathbf{u}_{j}^{k}\right) \mathbf{u}_{j}^{k+1}+\mathbf{g}\left(\mathbf{u}_{j}^{k}\right)
$$

with non-negative initial data. So, it is easy to see that all element of $\mathbf{u}_{j}^{k}$ is non-negative for all $j$ and $k$, provided if the linear stability condition $\Delta t / \Delta x^{2} \leq 1 /(2 d)$ for $d>0$ in the Lax-Richtmyer sense is assumed. Note that the similar scheme has also been introduced by Mimura in ( $[6],[7])$ for ensuring the postivity of numerical solutions, basically. In fact, Mimura argued the reaction diffusion equation of following type:

$$
\frac{\mathbf{u}_{j}^{k+1}-\mathbf{u}_{j}^{k}}{\Delta t}=\mathcal{D} \frac{\mathbf{u}_{j+1}^{k}-2 \mathbf{u}_{j}^{k}+\mathbf{u}_{j-1}^{k}}{\Delta x^{2}}+\widetilde{F}\left(\mathbf{u}_{j}^{k}\right) \mathbf{u}_{j}^{k+1}
$$

with non-negative diagonal matrix $\mathcal{D}$. From this procedure, we can also get positive solutions, under the linear stability conditions. However, it is not clear whether the invariant region for numerical solutions to (1) and (2) is derived, in general.

\section{Numerical Solutions to (BZ)}

We will derive invariant regions for numerical solutions to (BZ). For the sake of simplicity, let $n=1$, and let us consider (BZ) in bounded interval $x \in[0, L]$ for $L>0$ with the homogeneous Neumann boundary conditions $\partial_{x} u(0, t)=\partial_{x} u(L, t)=0$ or, the periodic boundary conditions $u(x, t)=u(x+L, t)$ for $t>0$. For discretization of (BZ), we put $u_{j}^{k} \approx u\left(x_{j}, t_{k}\right)$ and $v_{j}^{k} \approx v\left(x_{j}, t_{k}\right)$ for $j=0, \ldots, J$ and $k \in \mathbb{N}_{0}$, taking e.g. the average of integration. Here, $J \in \mathbb{N}, x_{j}:=j \Delta x$, $t_{k}:=k \Delta t$ for $\Delta x>0$ and $\Delta t>0 ; L=J \Delta x$.

We sometimes employ the algorithm of operator splitting methods (OSM) for solving the discretized reaction diffusion equation and related problems. For the discretization of uniform-in-space (BZ) with (DE) algorithm, let us consider

$$
\left(\mathrm{D}_{\mathrm{o}}\right) \quad\left\{\begin{array}{l}
\frac{u_{j}^{k+1}-u_{j}^{k}}{\Delta t}=\frac{u_{j}^{k}\left(1-u_{j}^{k+1}\right)}{\varepsilon}-h v_{j}^{k} \frac{u_{j}^{k+1}-q}{u_{j}^{k}+q}, \\
\frac{v_{j}^{k+1}-v_{j}^{k}}{\Delta t}=-v_{j}^{k+1}+u_{j}^{k}
\end{array}\right.
$$


for $j=1, \ldots, J-1$ and $k \in \mathbb{N}_{0}$. On the other hand, for the discretization of the heat equations, we use the standard FTCS (forward difference for time and second-order central difference for space)

$$
\left\{\begin{array}{l}
\frac{\tilde{u}_{j}^{k+1}-\tilde{u}_{j}^{k}}{\Delta t}=\frac{\tilde{u}_{j+1}^{k}-2 \tilde{u}_{j}^{k}+\tilde{u}_{j-1}^{k}}{\Delta x^{2}}, \\
\frac{\tilde{v}_{j}^{k+1}-\tilde{v}_{j}^{k}}{\Delta t}=d \frac{\tilde{v}_{j+1}^{k}-2 \tilde{v}_{j}^{k}+\tilde{v}_{j-1}^{k}}{\Delta x^{2}}
\end{array}\right.
$$

for $j=1, \ldots, J-1$ and $t \in \mathbb{N}_{0}$; at $j=0$ and $j=J$, we give certain definition by boundary conditions. Our algorithm is to solve alternate $\left(\mathrm{D}_{\mathrm{o}}\right)$ and $\left(\mathrm{D}_{\mathrm{h}}\right)$. That is to say, a pair of the series $\left\{u_{j}^{k}, v_{j}^{k}\right\}$ is given as

(i) Put $u_{j}^{0} \approx u_{0}\left(x_{j}\right)$ and $v_{j}^{0} \approx v_{0}\left(x_{j}\right)$, the average of integration.

(ii) Construct $u_{j}^{1}, v_{j}^{1}$ by $\left(\mathrm{D}_{\mathrm{o}}\right)$ with $k=0$.

(iii) Construct $\tilde{u}_{j}^{1}, \tilde{v}_{j}^{1}$ by $\left(\mathrm{D}_{\mathrm{h}}\right)$ with $\tilde{u}_{j}^{0}:=u_{j}^{1}$ and $\tilde{v}_{j}^{0}:=v_{j}^{1}$.

(iv) Construct $u_{j}^{2}, v_{j}^{2}$ by $\left(\mathrm{D}_{\mathrm{o}}\right)$ with $u_{j}^{1}:=\tilde{u}_{j}^{1}$ and $v_{j}^{1}:=\tilde{v}_{j}^{1}$.

(v) Construct $\tilde{u}_{j}^{2}, \tilde{v}_{j}^{2}$ by $\left(\mathrm{D}_{\mathrm{h}}\right)$ with $\tilde{u}_{j}^{1}:=u_{j}^{2}$ and $\tilde{v}_{j}^{1}:=v_{j}^{2}$.

(vi) Repeat this process.

If $d=0$, then we skip the steps of construction $\tilde{v}_{j}^{k}$, that is, $\tilde{v}_{j}^{k}:=v_{j}^{k}$. We will state the results on numerical solutions to discretized (BZ).

Theorem 3. Let $\varepsilon, h, \Delta t, \Delta x>0, d \geq 0$ and $q \in(0,1)$. Define $u_{j}^{k}, v_{j}^{k}$ as numerical solutions to alternate $\left(\mathrm{D}_{\mathrm{o}}\right)$ and $\left(\mathrm{D}_{\mathrm{h}}\right)$. If $u_{j}^{0}, v_{j}^{0} \in(q, 1)$ for $j$, then $u_{j}^{k}, v_{j}^{0} \in(q, 1)$ for $j$ and $k$, provided if $\Delta t / \Delta x^{2} \leq 1 / \max \{2,2 d\}$.

Proof. By Theorem 2 and the linear stability conditions, it holds that $u_{j}^{k}, v_{j}^{k} \geq 0$ for all $j$ and $k$. The induction in $k$ is used. Let $u_{j}^{k}, v_{j}^{k} \in$ $(q, 1)$. We first check that $u_{j}^{k+1}, v_{j}^{k+1}>q$ by $\left(\mathrm{D}_{\mathrm{o}}\right)$. It turns out that

$$
\begin{aligned}
u_{j}^{k+1}-q & =\frac{u_{j}^{k}+u_{j}^{k} \Delta t / \varepsilon+h q v_{j}^{k} \Delta t /\left(u_{j}^{k}+q\right)}{1+u_{j}^{k} \Delta t / \varepsilon+h v_{j}^{k} \Delta t /\left(u_{j}^{k}+q\right)}-q \\
& =\frac{\left(u_{j}^{k}-q\right)+(1-q) u_{j}^{k} \Delta t / \varepsilon}{1+u_{j}^{k} \Delta t / \varepsilon+h v_{j}^{k} \Delta t /\left(u_{j}^{k}+q\right)}>0
\end{aligned}
$$

by $u_{j}^{k}>q$ and $q \in(0,1)$. Similarly, we have

$$
v_{j}^{k+1}-q=\frac{v_{j}^{k}+u_{j}^{k} \Delta t}{1+\Delta t}-q=\frac{\left(v_{j}^{k}-q\right)+\left(u_{j}^{k}-q\right) \Delta t}{1+\Delta t}>0 .
$$

One can also easily see that

$$
\begin{aligned}
& 1-u_{j}^{k+1}=\frac{\left(1-u_{j}^{k}\right)+(1-q) h v_{j}^{k} \Delta t /\left(u_{j}^{k}+q\right)}{1+u_{j}^{k} \Delta t / \varepsilon+h v_{j}^{k} \Delta t /\left(u_{j}^{k}+q\right)}>0, \\
& 1-v_{j}^{k+1}=\frac{\left(1-v_{j}^{k}\right)+\left(1-u_{j}^{k}\right) \Delta t}{1+\Delta t}>0 .
\end{aligned}
$$

On $\left(\mathrm{D}_{\mathrm{h}}\right)$, it is well-known that the linear stability condition yields the maximum principle for numerical solution, that is, $\tilde{u}_{j}^{k+1}, \tilde{v}_{j}^{k+1} \in$ $(q, 1)$, if $\tilde{u}_{j}^{k}, \tilde{v}_{j}^{k} \in(q, 1)$. This completes the proof. 
Remark. (i) This assertion implies that $S_{\Delta}:=(q, 1)^{2}$ is an invariant region for numerical solutions to alternate $\left(D_{o}\right)$ and $\left(D_{h}\right)$.

(ii) One can easily see that $\mathbb{R}_{+}^{2}$ is also an invariant region by positivitypreserving.

(iii) The numerical solutions converge to solutions to PDE (BZ) as $\Delta t \rightarrow 0$ with order $O(\Delta t)$, as the same as the standard scheme and equation (2).

(iv) The similar results on the predator-prey models are also obtained.

The reader can find the details on PDE in [9] and references therein.

(v) The authors believe that one may take initial data, more freely.

In fact, if $u_{j}^{0}, v_{j}^{0} \geq 0$ for $j$, and if $u_{j^{\prime}}^{0}, v_{j^{\prime \prime}}^{0}>0$ for some $j^{\prime}$ and $j^{\prime \prime}$, then there exists a $k^{\prime} \in \mathbb{N}_{0}$ such that $u_{j}^{k}, v_{j}^{k} \in(q, 1)$ for $k \geq k^{\prime}$ and $j$. This means that absorbing sets for numerical solutions always exist in $S_{\Delta}$.

\section{Conclusion}

The positivity of solution for BZ is ensured by discretization scheme of reaction diffusion equation written as $\left(D_{o}\right)$ and $\left(D_{h}\right)$. The difference equations of discretized (BZ) with special type present the similar results to the unique smooth solutions of differential equations (BZ).

\section{Acknowledgements}

The authors would like to express their gratitude to Professor Hiroyuki Usami, Professor Shinya Miyajima and Professor Shintaro Kondo for letting them know some results on ordinary differential equations and numerical methods. The authors also like to express their gratitude to Professor Yoshihisa Morita for letting them know the results by Mimura and his collaborators ( [6], [7]).

\section{Conflicts of Interest}

The authors declare no conflicts of interest regarding the publication of this paper.

\section{References}

[1] Chen, G. (2000) A Mathematical Model for Bifurcations in a Belousov-Zhabotinsky Reaction. Physica D: Nonlinear Phenomena, 145, 309-329. https://doi.org/10.1016/S0167-2789(00)00113-5

[2] Keener, J.P. and Tyson, J.J. (1986) Spiral Waves in the BelousovZhabotinskii Reaction. Physica D: Nonlinear Phenomena, 21, 307324. https://doi.org/10.1016/0167-2789(86)90007-2

[3] Kondo, S., Novrianti, N., Sawada, O. and Tsuge, N. (2020) A Well-Posedness for the Reaction Diffusion Equations of BelousovZhabotinsky Reaction. To appear in Osaka J. Math.

[4] Furihata, D. and Matsuo, T. (2011) Discrete Variational Derivative Method. A Structure-Preserving Numerical Method for Partial Differential Equations. CRC Press, Boca Raton, FL. https://doi.org/10.1201/b10387

[5] Yun, A., Jeong, D. and Kim, J. (2012) An Efficient and Accurate Numerical Scheme for Turing Instability on a Predator-Prey Mod- 
el. International Journal of Bifurcation and Chaos, 22, Article ID: 1250139. https://doi.org/10.1142/S0218127412501398

[6] Mimura, M., Kametaka, Y. and Yamaguti, M. (1971) On a Certain Difference Scheme for Some Semilinear Diffusion System. Proceedings of the Japan Academy, 47, 385-387. https://doi.org/10.3792/pja/1195519973

[7] Mimura, M. and Nakaoka, A. (1972) On Some Degenerate Diffusion System Related with a Certain Reaction System. Journal of Mathematics of Kyoto University, 12, 95-121.

https://doi.org/10.1215/kjm/1250523562

[8] Tanaka, M., Nagahara, H., Kitahata, H., Krinsky, V., Agladze, K. and Yoshikawa, K. (2007) Survival versus Collapse: Abrupt Drop of Excitability Kills the Traveling Pulse, While Gradual Change Results in Adaptation. Physical Review E, 76, Article ID: 016205. https://doi.org/10.1103/PhysRevE.76.016205

[9] Novrianti, N., Sawada, O. and Tsuge, N. (2020) Positive Solutions to the Reaction Diffusion Equations for Prey-Predator Model with Dormancy of Predators. Preprint. 